\title{
On structure of effective action in four-dimensional quantum dilaton supergravity*
}

TSPU-TH34/96

\author{
I.L.Buchbinder ${ }^{\dagger}$ and A.Yu.Petrov \\ Department of Theoretical Physics \\ Tomsk State Pedagogical University \\ Tomsk 634041, Russia
}

Short title: Effective action for dilaton supergravity

PACS numbers: 04.60, 04.62, 11.30

* to be published in Classical and Quantum Gravity

${ }^{\dagger}$ e-mail:josephb@tspi.tomsk.su 


\begin{abstract}
A general structure of effective action in new chiral superfield model associated with $N=1, D=4$ supergravity is investigated. This model corresponds to finite quantum field theory and does not demand the regularization and renormalization at effective action calculation. It is shown that in local approximation the effective action is defined by two objects called general superfield effective lagrangian and chiral superfield effective lagrangian. A proper-time method is generalized for calculation of these two effective lagrangians in superfield manner. Power expansion of the effective action in supercovariant derivatives is formulated and the lower terms of such an expansion are calculated in explicit superfield form.
\end{abstract}




\section{Introduction}

In our previous paper [1] we have presented a new model of chiral superfield and investigated its quantum formulation. This model is conditioned by supertrace anomaly of the matter superfields in external superfield of $N=1, D=4$ supergravity. The corresponding anomaly generating action has been constructed in ref.[2]. We have considered a theory, action of which is a sum of the anomaly generating action and the action of supergravity. Being transformed to the conformally flat superspace this action takes a form of action of some chiral-antichiral model in flat superspace.

The model under consideration possesses the remarkable properties in infrared domain. This model is renormalizable, it is infrared free in some of couplings and it is finite in infrared limit. It means that the model can be considered as a natural infrared limit of quantum supergravity with matter.

Our goal in the present paper is to investigate a structure of the effective action of the above model in infrared domain. Aspects of effective action in supersymmetric models have been studied in a number of papers [3-12]. Đ The feature of our model is that the model is finite. Therefore we face a problem of effective action in a finite quantum field theory and should expect an appearance of the new aspects.

In the recent papers [3,4,8-10] (see also the book [5]) the method of calculation of effective action in superfield theories has been developed and its applications to one- and two-loop superfield effective potentials in Wess- Zumino model have been considered. This method is based on superfield form of proper-time technique and provides a simple enough and manifestly supersymmetric procedure of finding the effective action. In this paper we generalize the method introduced and used in refs. $[3,4,8-10]$ in order to take into account the features of the dilaton quantum supergravity model.

The essential feature of the model under consideration is a presence of higher derivatives 2]. Therefore we should solve a problem of development of superfield proper-time technique for higher derivative theories.

In any supersymmetric model containing the chiral and antichiral superfields a corresponding effective lagrangian is represented as a sum of two contributions. One of them depends only on chiral superfields and their space-time derivatives and can be called a chiral effective lagrangian. Another term depends both on chiral and antichiral superfields and their supercovariant derivatives and can be called a general effective lagrangian. Of course, an exact calculation of chiral and general effective lagrangians is impossible and we have to use the approximate schemes. It is natural to search the effective lagrangians in form of power expansion in supercovariant derivatives. As we will see the remarkable properties of the model under consideration stipulated by its finiteness allow to write down each term in these expansions in explicit form up to some constants. In particular, here we can not expect in the effective lagrangians any logarithmic contributions which are most typical in

\footnotetext{
${ }^{1}$ We would like to notice that some classical aspects of the [11] have also been considered in earlier paper 23]

${ }^{2}$ We do not discuss here the question of unitarity of $S$-matrix, caused by higher derivatives. The model under consideration should be understood as an effective theory and aimed for investigation of low-energy behaviour.
} 
the standard quantum field theories with divergences. As a result we have the only problem how to evaluate above constants.

Use of the superfield proper-time technique allows to calculate any term in above expansions for one-loop general and chiral effective lagrangians. In this paper we find the lower terms in these expansions and show they have no any divergences and their structure corresponds to result of general analysis of the effective lagrangian on the base of the dimension and finiteness of the theory.

The paper is organized as follows. The section 2 is devoted to description of the model, definition of superfield effective actions and its symmetries and investigating the properties of the general and chiral lagrangians. In section 3 the method of calculating the one-loop effective action is developed. In particular we show that the one-loop effective action for chiral- antichiral superfield model can be expressed in terms of some vector multiplet model. The sections 4 and 5 are devoted to calculating the lower terms in expansions of general and chiral one-loop lagrangians in derivatives. In section 6 we show the correspondense between the model under consideration and massless Wess-Zumino model. The summary is devoted to general discussion of the results.

\section{Structure of effective action}

The previous paper [1] has been devoted to investigation of structure of renormalization and infrared asymptotical behaviour of four-dimensional dilaton supergravity model. The action of this model is given as a sum of the action generating the superconformal anomaly and the classical action of $N=1$ supergravity. The complete action in the conformally flat superspace has the form

$$
\begin{aligned}
S & =\int d^{8} z\left(-\frac{Q^{2}}{2(4 \pi)^{2}} \bar{\sigma} \square \sigma+\bar{D}^{\dot{\alpha}} \bar{\sigma} D^{\alpha} \sigma \times\left(\xi_{1} \partial_{\alpha \dot{\alpha}}(\sigma+\bar{\sigma})+\right.\right. \\
& \left.\left.+\xi_{2} \bar{D}_{\dot{\alpha}} \bar{\sigma} D_{\alpha} \sigma\right)-\frac{m^{2}}{2} e^{\sigma+\bar{\sigma}}\right)+\left(\Lambda \int d^{6} z e^{3 \sigma}+\text { h.c. }\right)
\end{aligned}
$$

Here $\sigma=\ln \Phi$ where $\Phi$ is a chiral supergravity compensator (the only dynamical field in this theory), $Q^{2}, \xi_{1}, \xi_{2}, m^{2}, \Lambda$ are couplings. $\sigma$ is dimensionless.

We have investigated renormalization group equations for the effective couplings of this theory and proved that in infrared limit (i.e at $t \rightarrow-\infty$ ) they satisfy the conditions: $\xi_{1}(t)=\xi_{2}(t)=m^{2}(t)=0, Q^{2}(t)=Q^{2} \neq 0$. So, action of the theory in infrared domain can be written in the form

$$
S=\int d^{8} z\left(-\frac{1}{2} \frac{Q^{2}}{(4 \pi)^{2}} \bar{\sigma} \square \sigma\right)+\left(\Lambda \int d^{6} z e^{3 \sigma}+\text { h.c. }\right)
$$

It is easy to show that classical action (2) is invariant under transformations

$$
\begin{array}{r}
\sigma \rightarrow \sigma+\alpha, \bar{\sigma} \rightarrow \bar{\sigma}+\beta \\
\Lambda \rightarrow e^{-3 \alpha} \Lambda, \bar{\Lambda} \rightarrow e^{-3 \beta} \Lambda
\end{array}
$$

$\alpha$ and $\beta$ are the independent parameters, for example, for chiral effective action

independent on $\bar{\sigma}$ we put $\beta=0$ and $\Lambda$ is not transformed. After introduction of 
classical background field $\sigma$ and the quantum one $\chi$ [13] we arrive at the following quantum action for $\chi$ which will be also invariant under transformations (3) of background superfields $\sigma$ and $\bar{\sigma}$.

$$
S_{q}=\int d^{8} z\left(-\frac{Q^{2}}{2(4 \pi)^{2}} \bar{\chi} \square \chi\right)+\left(\Lambda \int d^{6} z e^{3 \sigma}\left(e^{3 \chi}-1-3 \chi\right)+\text { h.c. }\right)
$$

The quantum field $\chi$ in (4) is not transformed under these transformations (3).

The effective action of the theory has the form

$$
\Gamma[\sigma, \bar{\sigma}]=S[\sigma, \bar{\sigma}]+\bar{\Gamma}[\sigma, \bar{\sigma}]
$$

where $S[\sigma, \bar{\sigma}]$ is the classical action (2) and $\bar{\Gamma}[\sigma, \bar{\sigma}]$ is the sum of all quantum corrections. Here the functional $\bar{\Gamma}[\sigma, \bar{\sigma}]$ is defined as follows (see f.e. [13])

$$
\exp (i \bar{\Gamma}[\sigma, \bar{\sigma}])=\int D \chi D \bar{\chi} \exp \left(i S_{q}[\sigma, \bar{\sigma} ; \chi, \bar{\chi}]\right)
$$

It was proved in the previous paper [1] that the theory under consideration is finite so any anomalies in this theory cannot arise. Therefore we should expect that the effective action is also invariant under the transformations (3).

We suppose that the quantum correction to the effective action can be rewritten as

$$
\bar{\Gamma}=\int d^{8} z L+\left(\int d^{6} z \mathcal{L}_{c}+\text { h.c. }\right)
$$

Here $L$ is a general effective lagrangian, $\mathcal{L}_{c}$ is a chiral effective lagrangian satisfying the condition $\bar{D}_{\dot{\alpha}} \mathcal{L}_{c}=0$ i.e. $\mathcal{L}_{c}$ is a chiral superfield itself. $\mathcal{L}_{c}(\sigma)$ depends on $\sigma$ and its space-time derivatives but not on $D_{\alpha} \sigma, D^{2} \sigma$. One can suppose that $\mathcal{L}_{c}(\sigma)$ can depend on $\bar{D}^{2} \bar{\sigma}$ since $\bar{D}^{2} \bar{\sigma}$ is a chiral superfield, i.e. in the expansion of $\mathcal{L}_{c}(\sigma)$ the terms of the form $f\left(\sigma, \partial \sigma, \partial^{2} \sigma \ldots\right)\left(\bar{D}^{2} \bar{\sigma}\right)^{n}$ formally can arise but their contribution to the effective action of the form $\int d^{6} z f\left(\sigma, \partial \sigma, \partial^{2} \sigma \ldots\right)\left(\bar{D}^{2} \bar{\sigma}\right)^{n}$ can be rewritten as an integral over whole superspace as $\int d^{8} z f\left(\sigma, \partial \sigma, \partial^{2} \sigma \ldots\right)\left(\bar{D}^{2} \bar{\sigma}\right)^{n-1}$ It means we can consider such terms as the corrections to the general effective lagrangian and put $\mathcal{L}_{c}(\sigma)$ to be independent on $\bar{\sigma}$.

The effective lagrangians $L(\sigma, \bar{\sigma})$ and $\mathcal{L}_{c}(\sigma)$ can be represented in the form of power expansion in derivatives of $\sigma, \bar{\sigma}$. The lower correction to $L$ should has the form $L \sim \Lambda^{k_{1}} \bar{\Lambda}^{k_{2}} e^{n(\sigma+\bar{\sigma})}$ where $k_{1}, k_{2}$ and $n$ are some numbers. It is turned out that all these numbers, in principle, can be found exactly without any calculations. Namely, requirements that the dimension of $L$ is 2 and that the action is invariant under the transformations (3) allow to obtain the lower contribution to general effective lagrangian in the form

$$
K=\rho(\Lambda \bar{\Lambda})^{1 / 3} e^{\sigma+\bar{\sigma}}
$$

where $\rho$ is some dimensionless constant which can be found only at straightforward calculations.

The lower contribution to $\mathcal{L}_{c}$ should have the form of a linear combination of the terms

$\Lambda^{q_{1}} \bar{\Lambda}^{q_{2}} e^{p \sigma} \partial_{m} \sigma \partial^{m} \sigma$ and $\Lambda^{q_{1}} \bar{\Lambda}^{q_{2}} e^{p \sigma} \square \sigma$ where $q_{1}, q_{2}, p$ are some numbers. The dimension of $\mathcal{L}_{c}$ is 3 , hence $3\left(q_{1}+q_{2}\right)+2=3$. The invariance of $\mathcal{L}_{c}$ under above 
transformations leads to $-3 q_{1}+p=0$ (we use transformations at $\beta=0$ there). As a result $q_{1}=p / 3, q_{2}=1 / 3-p / 3$. Hence the lower contribution to $\mathcal{L}_{c}$ looks like this

$$
\mathcal{L}_{c}=\bar{\Lambda}^{1 / 3}\left[\sum_{p_{1}}\left(\frac{\Lambda}{\bar{\Lambda}}\right)^{p_{1}} \rho_{p_{1}} e^{p_{1} \sigma} \partial_{m} \sigma \partial^{m} \sigma+\sum_{p_{2}}\left(\frac{\Lambda}{\bar{\Lambda}}\right)^{p_{2}} \rho_{p_{2}} e^{p_{2} \sigma} \square \sigma\right]
$$

where $\rho_{p_{1}}$ and $\rho_{p_{2}}$ are some dimensionless constants. These constants and the values of $p_{1}$ and $p_{2}$ can be found at straightforward calculations.

In this paper we will calculate the lower corrections to the general effective lagrangian $L$ and to chiral effective lagrangian $\mathcal{L}_{c}(\sigma)$ at the one-loop level exactly.

\section{Problems of calculating the one-loop effective action}

It is well known that the one-loop correction to the effective action $\bar{\Gamma}^{(1)}$ has the form

$$
\exp \left(i \bar{\Gamma}^{(1)}\right)=\int D \chi D \bar{\chi} \exp \left(i S_{q}^{(2)}[\sigma, \bar{\sigma} ; \chi, \bar{\chi}]\right)
$$

where $S_{q}^{(2)}$ is a quadratic part of $S_{q}(4)$. In the case under consideration one can obtain

$$
\begin{aligned}
\exp \left(i \bar{\Gamma}^{(1)}\right) & =\int D \chi D \bar{\chi} \exp \left\{\left[\int d^{8} z\left(-\frac{1}{2} \frac{Q^{2}}{(4 \pi)^{2}} \bar{\chi} \square\right)+\right.\right. \\
& \left.\left.+\left(\Lambda \int d^{6} z e^{3 \sigma} \frac{(3 \chi)^{2}}{2}+\text { h.c. }\right)\right]\right\}=\operatorname{Det}^{-1 / 2} \mathcal{H}
\end{aligned}
$$

where

$$
\mathcal{H}=\left(\begin{array}{cc}
9 \Lambda e^{3 \sigma} & \square \frac{\bar{D}^{2}}{4} \\
\square \frac{D^{2}}{4} & 9 \bar{\Lambda} e^{3 \bar{\sigma}}
\end{array}\right)
$$

Straightforward calculating Det $\mathcal{H}$ is a very complicated problem since elements of this matrix are defined in different subspaces of the superspace and mix the chiralities. In the papers $[3,4]$ where the Wess-Zumino model was investigated a trick allowing to avoid these difficulties has been suggested and the effective action has been expressed in terms of Green function of the real scalar superfield. We will show that the trick carried out in these papers can be applied to the theory under consideration too.

We consider a theory of a real scalar superfield with the action

$$
S=-\frac{Q^{2}}{(4 \pi)^{2}} \frac{1}{16} \int d^{8} z V D^{\alpha} \bar{D}^{2} D_{\alpha} \square V
$$

and calculate a corresponding one-loop effective action.

This theory is invariant under gauge transformations

$$
V \rightarrow V^{\Psi}=V+i(\bar{\Psi}-\Psi), \bar{D}_{\dot{\alpha}} \Psi=0, D_{\alpha} \bar{\Psi}=0
$$


We choose the gauge-fixing functions as follows

$$
\begin{aligned}
& F(V)=\frac{1}{4} \bar{D}^{2} V-\chi \\
& \bar{F}(V)=\frac{1}{4} D^{2} V-\bar{\chi}
\end{aligned}
$$

Their variation under transformations (13) has the form

$$
\delta\left(\begin{array}{c}
F(V) \\
\bar{F}(V)
\end{array}\right)=-M^{(0)}\left(\begin{array}{c}
i \Psi \\
i \bar{\Psi}
\end{array}\right)
$$

where

$$
M^{(0)}=\left(\begin{array}{cc}
0 & -\frac{1}{4} \bar{D}^{2} \\
-\frac{1}{4} D^{2} & 0
\end{array}\right)
$$

As a result we can write

$$
\operatorname{det} M^{(0)} \int D \Psi D \bar{\Psi} \delta_{+}\left(F\left(V^{\Psi}\right)\right) \delta_{-}\left(\bar{F}\left(V^{\Psi}\right)\right)=1
$$

The one-loop effective action $W_{v}$ for the theory (12) has the form

$$
\begin{aligned}
\exp \left(i W_{v}\right) & =\int D V \operatorname{det} M^{(0)} \delta_{+}(F(V)) \delta_{-}(\bar{F}(V)) \exp \left\{-\frac{1}{16} \frac{Q^{2}}{(4 \pi)^{2}} \times\right. \\
& \left.\times \int d^{8} z V D^{\alpha} \bar{D}^{2} D_{\alpha} \square V\right\}
\end{aligned}
$$

By definition $\exp \left(i W_{v}\right)$ is a constant. So, after multiplying (11) and (19) we obtain

$$
\begin{aligned}
\exp \left(i \bar{\Gamma}^{(1)}+i W_{v}\right) & =\int D \chi D \bar{\chi} D V \operatorname{det} M^{(0)} \delta_{+}\left(\frac{1}{4} \bar{D}^{2} V-\chi\right) \delta_{-}\left(\frac{1}{4} \bar{D}^{2} V-\chi\right) \times \\
& \times \exp \left\{-\frac{1}{16} \frac{Q^{2}}{(4 \pi)^{2}} \int d^{8} z V D^{\alpha} \bar{D}^{2} D_{\alpha} \square V\right\}- \\
& -\left\{\left[\int d^{8} z\left(-\frac{1}{2} \frac{Q^{2}}{(4 \pi)^{2}} \bar{\chi} \square \chi\right)+\left(\Lambda \int d^{6} z e^{3 \sigma} \frac{(3 \chi)^{2}}{2}+\text { h.c. }\right)\right]\right\}
\end{aligned}
$$

Let us integrate over $\chi$ using delta-functions, then one obtains

$$
\begin{aligned}
& \exp \left(i \bar{\Gamma}^{(1)}\right) \exp \left(i W_{v}\right) d e t^{-1} M^{(0)}= \\
= & D V \exp \left\{\frac{1}{2} \int d^{8} z \frac{Q^{2}}{(4 \pi)^{2}} V \square^{2} V+\left(\frac{9 \Lambda}{2} \int d^{6} z e^{3 \sigma}\left(\frac{\bar{D}^{2} V}{4}\right)^{2}+\right.\right. \\
+ & \left.\left.\frac{9 \bar{\Lambda}}{2} \int d^{6} z e^{3 \bar{\sigma}}\left(\frac{D^{2} V}{4}\right)\right)\right\}=\int D V \exp \left(\frac{i}{2} V \Delta V\right)
\end{aligned}
$$

Since $W_{v}$ and $\operatorname{det} M^{(0)}$ are constants by the definitions we can obtain one-loop effective action for the theory under consideration in the form

$$
\bar{\Gamma}^{(1)}=\frac{i}{2} \operatorname{Tr} \ln \Delta
$$


where

$$
\Delta=\frac{Q^{2}}{(4 \pi)^{2}} \square^{2}-9 \Lambda e^{3 \sigma} \frac{\bar{D}^{2}}{4}-9 \bar{\Lambda} e^{3 \bar{\sigma}} \frac{D^{2}}{4}
$$

We would like to notice that the operator $\Delta$ contains the higher derivatives (it contains the four derivatives being written in component form).

From (22) we obtain

$$
\bar{\Gamma}^{(1)}=-\frac{i}{2} \int_{0}^{\infty} \frac{d s}{s} \operatorname{Tr} e^{i s \Delta}
$$

Our aim is to calculate $\Omega(\sigma, \bar{\sigma} \mid s)=e^{i s \Delta}$. Now let us consider general effective lagrangian and chiral effective lagrangian separately.

\section{General effective lagrangian}

We start with calculation of the lower correction to general effective lagrangian, i.e. a case when the fields $\sigma, \bar{\sigma}$ and hence $\Omega(\sigma, \bar{\sigma} \mid s)$ do not depend on derivatives of the fields. It is easy to see that the $\Omega$ has the form

$$
\begin{aligned}
\Omega(\sigma, \bar{\sigma} \mid s) & =\exp \left(i s\left(\frac{Q^{2}}{(4 \pi)^{2}} \square^{2}-\frac{9}{4} \Lambda e^{3 \sigma} \bar{D}^{2}-\frac{9}{4} \bar{\Lambda} e^{3 \bar{\sigma}} D^{2}\right)\right)= \\
& =\exp \left(i s\left(-\frac{9}{4} \Lambda e^{3 \sigma} \bar{D}^{2}-\frac{9}{4} \bar{\Lambda} e^{3 \bar{\sigma}} D^{2}\right)\right) \exp \left(i s \frac{Q^{2}}{(4 \pi)^{2}} \square^{2}\right)
\end{aligned}
$$

Hence, to calculate a trace of $\Omega$ we must compute:

$$
\begin{aligned}
& \text { 1. } \tilde{\Omega}=\exp \left(i s\left(-\frac{9}{4} \Lambda e^{3 \sigma} \bar{D}^{2}-\frac{9}{4} \bar{\Lambda} e^{3 \bar{\sigma}} D^{2}\right)\right) \\
& \text { 2. } U\left(x, x^{\prime} \mid s\right)=\exp \left(i s \frac{Q^{2}}{(4 \pi)^{2}} \square^{2}\right) \delta^{4}\left(x-x^{\prime}\right)
\end{aligned}
$$

For simplifying calculations we will introduce a new parameter $s$ using a rule $s \frac{Q^{2}}{(4 \pi)^{2}} \rightarrow s$ and denote

$$
h=-\frac{9}{4} \Lambda \frac{(4 \pi)^{2}}{Q^{2}}, \bar{h}=-\frac{9}{4} \bar{\Lambda} \frac{(4 \pi)^{2}}{Q^{2}}, \varphi=e^{3 \sigma}, \bar{\varphi}=e^{3 \bar{\sigma}}
$$

Then $\Omega$ has the form

$$
\Omega(\sigma, \bar{\sigma} \mid s)=\exp \left(i s\left(\square^{2}+h \varphi \bar{D}^{2}+\bar{h} \bar{\varphi} D^{2}\right)\right)=\exp \left(i s\left(h \varphi \bar{D}^{2}+\bar{h} \bar{\varphi} D^{2}\right)\right) \exp \left(i s \square^{2}\right)
$$

Let us consider at first the function $\tilde{\Omega}=\exp \left(i s\left(h \varphi \bar{D}^{2}+\bar{h} \bar{\varphi} D^{2}\right)\right)$ satisfying the equation

$$
i \frac{\partial \tilde{\Omega}}{\partial s}=-\tilde{\Omega} \tilde{\Delta}
$$

Here

$$
\tilde{\Delta}=h \varphi \bar{D}^{2}+\bar{h} \bar{\varphi} D^{2},\left.\tilde{\Omega}\right|_{s=0}=1
$$


Let us represent $\tilde{\Omega}$ in the form of expansion in spinor supercovariant derivatives [3], [4]:

$$
\begin{array}{r}
\tilde{\Omega}=1+\frac{1}{16} A(\tilde{s}) \bar{D}^{2} D^{2}+\frac{1}{16} \tilde{A}(\tilde{s}) D^{2} \bar{D}^{2}+\frac{1}{8} B^{\alpha}(\tilde{s}) D_{\alpha} \bar{D}^{2}+\tilde{B}_{\dot{\alpha}}(\tilde{s}) \bar{D}^{\dot{\alpha}} D^{2}+ \\
\frac{1}{4} C(\tilde{s}) D^{2}+\frac{1}{4} \tilde{C}(\tilde{s}) \bar{D}^{2}
\end{array}
$$

The solution of the eq. (27) for $\tilde{\Omega}(28)$ with the initial conditions: $A, \tilde{A}, B, \tilde{B}, C, \tilde{C}$ at $s=0$ are equal to zero looks like this

$$
A=\tilde{A}=\frac{1}{\square}[\cosh (4 \tilde{s} \sqrt{h \bar{h} \varphi \bar{\varphi} \square})-1]
$$

where $\tilde{s}=-i s$ (see some details in refs. [3,4]). It follows from $(24,28)$ that only terms containing $A$ and $\tilde{A}$ give non-zero contribution when calculating trace of $\Omega$. The eq. (29) shows that lower contribution to one-loop general effective lagrangian depends only on $\varphi \bar{\varphi}=e^{3(\sigma+\bar{\sigma})}$.

After calculations described in Appendix A we obtain the lower contribution to one-loop general effective lagrangian in the form

$$
K^{(1)}=\left(\frac{c}{Q^{4 / 3}}\right)(\Lambda \bar{\Lambda})^{1 / 3} e^{\sigma+\bar{\sigma}}
$$

where $c$ is a finite constant. The explicit form for $c$ is given in Appendix A.

This result coincides with the general structure of $K$ obtained in the section 2 . We also see that although there is no the 'massive' term like $e^{\sigma+\bar{\sigma}}$ in this theory at a classical level such a term arises as a quantum correction. We can conclude that the lower contribution to one-loop general effective lagrangian is finite in correspondence with the results of the previous paper [1].

\section{Chiral effective lagrangian}

In this section we consider calculating the lower contribution to one-loop chiral effective lagrangian $\mathcal{L}_{c}^{(1)}$ i.e the part of one-loop effective action which is a chiral superfield itself and does not depend on $\bar{\sigma}$. To calculate the chiral effective lagrangian in the model under consideration one should put $\bar{\sigma}=0$ in the operator $\Omega(\sigma, \bar{\sigma})$ introduced in the section 3. Here and further we will denote $\Omega(\sigma)=\left.\Omega(\sigma, \bar{\sigma})\right|_{\bar{\sigma}=0}$. The operator $\Omega(\sigma)$ has the form

$$
\Omega(\sigma)=e^{i s \Delta}=\exp \left(i s\left(\square^{2}+h \varphi \bar{D}^{2}+\bar{h} D^{2}\right)\right)
$$

The part of one-loop effective action depending only on the chiral superfield $\varphi$ can be written as

$$
\tilde{\Gamma}^{(1)}=-\frac{i}{2} \int_{0}^{\infty} \frac{d s}{s} \operatorname{Tr} e^{i s \Delta}
$$

where $\Delta=\square^{2}+h \varphi \bar{D}^{2}+\bar{h} D^{2}$. We will call the one-loop chiral effective lagrangian $\mathcal{L}_{c}^{(1)}$ that part of $\tilde{\Gamma}^{(1)}$ which is a chiral superfield itself and depends only on $\varphi$ and its space-time derivatives. 
We can represent $\mathcal{L}_{c}^{(1)}$ as a power expansion in space-time derivatives. Our aim here consists in calculating the lower terms in this expansion.

Let us consider $e^{i s \Delta}$ in details. We can write it in the form

$$
e^{i s \Delta}=e^{i s \square^{2}} e^{i s\left(h \varphi \bar{D}^{2}+\bar{h} D^{2}\right)} M
$$

where $M$ depends on different commutators of the form

$$
\left[\square^{2},\left[\ldots\left[h \varphi \bar{D}^{2}+\bar{h} D^{2},\left[\ldots\left[\square^{2}, h \varphi \bar{D}^{2}+\bar{h} D^{2}\right]\right]\right]\right]\right.
$$

and can be represented in the form $M=1+$ (commutators) since if all these commutators are equal to zero, $M$ will be evidently equal to 1 . Due to the cyclic property of trace one can write

$$
\operatorname{Tr} e^{i s \Delta}=\operatorname{Tr} e^{i s\left(h \varphi \bar{D}^{2}+\bar{h} D^{2}\right)} M e^{i s \square^{2}}
$$

Further, it should be noted that the lower commutator in $M$ of the form $\left[\square^{2}, h \varphi \bar{D}^{2}+\right.$ $\left.\bar{h} D^{2}\right]$, and hence higher commutators contained in $M$ depend at least on first spacetime derivative of $\varphi$ so $M$ can be written in the form:

$$
M=1+\partial_{m} \varphi a^{m}+\ldots
$$

where $a_{m}$ depends on $\varphi, D_{\alpha} \varphi, D^{2} \varphi$ and contains $\partial$ in different powers, dots denote all terms proportional to second and higher space- time derivatives of $\varphi$.

Then, after calculation $\tilde{\Omega}=e^{i s\left(h \varphi \bar{D}^{2}+\bar{h} D^{2}\right)}$ we will obtain the coefficient $A$ in the expansion (28) in the form

$$
A(s)=A_{1}\left(s \mid \varphi, \partial \varphi_{. .}\right)+D^{\alpha} \varphi D_{\alpha} \varphi A_{2}(s \mid \varphi, \partial \varphi . .)+D^{2} \varphi A_{3}(s \mid \varphi, \partial \varphi . .)
$$

and $\tilde{A}$ in the analogous form. Here dots denote higher space-time derivatives of $\varphi$ and powers of $\square$. Because of (A.1) the one-loop chiral effective lagrangian has the form

$$
\mathcal{L}_{c}^{(1)}=-\frac{i}{2}\left(-\frac{1}{4}\right) \int_{0}^{\infty} \frac{d s}{s} \bar{D}^{2}<A(\tilde{s})+\tilde{A}(\tilde{s})>\left.U\left(x, x^{\prime} ; s\right)\right|_{x=x^{\prime}}
$$

which can be rewritten as follows

$$
\begin{aligned}
\mathcal{L}_{c}^{(1)} & =2 i \int_{0}^{\infty} \frac{d s}{s}\left(\partial_{m} \varphi \partial^{m} \varphi\left(A_{2}(s \mid \varphi, \partial \varphi . .)+\tilde{A}_{2}(s \mid \varphi, \partial \varphi . .)\right)+\right. \\
& \left.+\square \varphi\left(A_{3}(s \mid \varphi, \partial \varphi . .)+\tilde{A}_{3}(s \mid \varphi, \partial \varphi . .)\right)\right)\left.U\left(x, x^{\prime} ; s\right)\right|_{x=x^{\prime}}
\end{aligned}
$$

So dependence of $A$ and $\tilde{A}$ on $\partial \varphi$ and higher space-time derivatives of $\varphi$ gives contribution only to third and higher orders in expansion of $\mathcal{L}_{c}^{(1)}$ in derivatives. It is evidently that taking into account the commutators containing in $M$ we will get the higher orders in derivatives by analogous reasons. Hence to calculate chiral effective action up to the second order in derivatives one should:

1. put $M$ in (33) to be equal to 1 , i.e. put

$$
e^{i s \Delta}=e^{i s \square^{2}} e^{i s\left(h \varphi \bar{D}^{2}+\bar{h} D^{2}\right)}
$$

2. When calculating $e^{i s\left(h \varphi \bar{D}^{2}+\bar{h} D^{2}\right)}$ put $\partial_{m} \varphi$ and higher space-time derivatives of $\varphi$ to be equal to zero. 
So we can represent $\Omega(\sigma)$ for calculating the chiral effective action in the form

$$
\Omega(\sigma)=\tilde{\Omega}(\sigma) e^{i s \square^{2}}
$$

where $\tilde{\Omega}(\sigma)=\exp \left(i s\left(h \varphi \bar{D}^{2}+\bar{h} D^{2}\right)\right)=\exp (i s \tilde{\Delta})$. Here $\tilde{\Omega}(\sigma)$ satisfies the equation:

$$
i \frac{\partial \tilde{\Omega}(\sigma)}{\partial s}=-\tilde{\Omega}(\sigma)\left(h \varphi \bar{D}^{2}+h D^{2}\right)
$$

$\tilde{\Omega}$ can be represented in the form (28). The initial conditions coincide with those one for the general effective potential, namely, $\tilde{\Omega}=1$ and $A, \tilde{A}, B, \tilde{B}, C, \tilde{C}$ all are equal to zero at $s=0$.

We can solve the system for $\tilde{\Omega}$ by the method analogous to one of refs. [3,4]. The operatoral coefficients $A, \tilde{A}$ necessary for our calculations have the form

$$
\begin{aligned}
\tilde{A} & =\frac{1}{\square}(\cosh (W \tilde{s})-1) \\
A & =-16 \bar{h}\left\{-8 h \varphi \tilde{s}^{2}+\frac{64 h \bar{h}}{W^{2}}\left(\frac{\sinh (W \tilde{s})}{W}-\tilde{s}\right) D^{2} \varphi+\right. \\
& \left.+2^{13} \frac{h^{2} \bar{h}^{2}}{W^{5}} D^{\alpha} \varphi D_{\alpha} \varphi[W \tilde{s} \cosh (W \tilde{s})-3 \sinh (W \tilde{s})+2 W \tilde{s}]\right\}
\end{aligned}
$$

where $W=16 \sqrt{h \bar{h} \varphi \square}$ and $\varphi=e^{3 \sigma}$.

After the calculations described in Appendix B one obtains the one-loop chiral effective action up to the second order in derivatives in the form (B.III). Restoring $\sigma$ in this expression using the definition $\varphi=e^{3 \sigma}$ we arrive at $\mathcal{L}_{c}^{(1)}$ in the form

$$
\begin{aligned}
\mathcal{L}_{c}^{(1)} & =\bar{\Lambda}^{1 / 3}\left\{\left(\left(c_{1}+3 c_{3}\right)\left(\frac{\bar{\Lambda}^{1}}{{ }^{1 / 3}}\right)^{-\sigma}+c_{2}\left(\frac{\bar{\Lambda}}{\Lambda}\right)^{-2 / 3} e^{2 \sigma}+3 c_{4}\left(\frac{\bar{\Lambda}_{\Lambda}}{\Lambda}\right)^{4 / 3} e^{-4 \sigma}\right) \times\right. \\
& \left.\times \partial^{m} \sigma \partial_{m} \sigma+\square \sigma\left(c_{3}\left(\frac{\bar{\Lambda}}{\Lambda}\right)^{1 / 3} e^{-\sigma}+c_{4}\left(\frac{\bar{\Lambda}}{\Lambda}\right)^{4 / 3} e^{-4 \sigma}\right)\right\}
\end{aligned}
$$

where $c_{1}, c_{2}, c_{3}, c_{4}$ are finite constants given in Appendix B.

The eq. (35) is the final result for the lower correction to the one-loop chiral effective action. We see that it is not equal to zero but essentially dependent on space-time derivatives. This result corresponds to the general structure of $\mathcal{L}_{c}^{(1)}$ obtained in the section 2. We also can conclude that it is finite in correspondence with the results of the previous paper [1].

\section{Generating of Wess-Zumino model}

We have calculated the lower correction to the one-loop effective action for the theory under consideration and proved that the effective action in this approximation has the form:

$$
\begin{aligned}
\Gamma & =\int d^{8} z\left(-\frac{Q^{2}}{(4 \pi)^{2}} \frac{1}{2} \bar{\sigma} \square \sigma\right)+\left(\Lambda \int d^{6} z e^{3 \sigma}+\text { h.c. }\right)+ \\
& +c\left(\frac{\Lambda}{Q^{2}}\right)^{2 / 3} \int d^{8} z e^{\sigma+\bar{\sigma}}+
\end{aligned}
$$




$$
\begin{aligned}
& +\left(\frac{\Lambda}{Q^{2}}\right)^{1 / 3}\left[\int d ^ { 6 } z \left\{\left(\left(c_{1}+3 c_{3}\right) e^{-\sigma}+c_{2} e^{2 \sigma}+3 c_{4} e^{-4 \sigma}\right) \partial^{m} \sigma \partial_{m} \sigma+\right.\right. \\
& \left.\left.+\square \sigma\left(c_{3} e^{-\sigma}+c_{4} e^{-4 \sigma}\right)\right\}+ \text { h.c. }\right]
\end{aligned}
$$

Here ones put $\bar{\Lambda}=\Lambda$. If we also put $\partial_{m} \sigma=0, \partial_{m} \bar{\sigma}=0$ (i.e. consider the superfields slowly varying in space-time) we will obtain the effective action in the form

$$
\Gamma=c\left(\frac{\Lambda}{Q^{2}}\right)^{2 / 3} \int d^{8} z e^{\sigma+\bar{\sigma}}+\left(\Lambda \int d^{6} z e^{3 \sigma}+\text { h.c. }\right)
$$

Let us denote $\phi=\sqrt{c}\left(\frac{\Lambda}{Q^{2}}\right)^{1 / 3} e^{\sigma}, \bar{\phi}=\sqrt{c}\left(\frac{\Lambda}{Q^{2}}\right)^{1 / 3} e^{\bar{\sigma}}$. Then the eq. (37) can be rewritten as follows

$$
\Gamma=\int d^{8} z \phi \bar{\phi}+\left(\lambda \int d^{6} z \phi^{3}+\text { h.c. }\right)
$$

where $\lambda=Q^{2} c^{-3 / 2}$. This action describes dynamics of a massless chiral superfield $\phi$ of dimension equal to 1 and completely coincides with the action of massless WessZumino model. So one can conclude that the four-dimensional model of quantum supergravity chiral compensator in infrared limit leads to the Wess-Zumino model.

We see also that $\Lambda$ is absent in the action (38), its role consists in transformation of dimensionless field $\Phi\left(\Phi=e^{\sigma}\right.$ is a chiral compensator $)$ to the field $\phi$ with dimension 1.

\section{Summary}

We have investigated a structure of effective action for the dilaton supergravity in the infrared domain where the model is finite. The effective action is defined by general effective lagrangian and chiral effective lagrangian. We have considered a generic form of lower contributions to these effective lagrangians within expansions in supercovariant derivatives and found that the finiteness of the model allows to write down the terms in above expansions up to some constants.

Generalized superfield proper-time technique has been developed and its application to calculating the one-loop effective lagrangians has been investigated. In particular, the lower contributions to the general effective lagrangian and chiral effective lagrangian have been calculated in explicit superfield form.

We have studied a structure of one-loop effective action constructed on the base of the lower contributions to the effective lagrangians for the superfields slowly varying in space-time. It is shown that in this limit the effective action is reduced to the action of standard massless Wess-Zumino model.

We would like to notice in conclusion that the non-supersymmetric model of dilaton gravity has been developed in ref. [14]. The structure of effective action in this model was considered in refs. [15-22]. The supersymmetric model studied here is finite unlike non-supersymmetric version. It leads to simple enough structure of the effective lagrangians in compare with one in non- supersymmetric model [15-22].

\section{Acknowledgements}

The authors are grateful to S.D.Odintsov and S.M.Kuzenko for interesting discussions on some aspects of the work. We thankful to S.J.Gates paying our attention 
to paper [23]. The work was supported in part by Russian Foundation for Basic Research under the project No. 94-02-03234. I.L.B. thanks D.Luest, D.Ebert and H.Dorn for their hospitality during his visit to Institute of Physics, Humboldt Berlin University where most part of the work has been fulfilled. This visit was supported by Deutsche Forschungsgemeinschaft under contract DFG-436 RUS 113. 


\section{Appendix A. Calculation of one-loop general effective po- tential}

It is well known from refs. [3,4] that the one-loop effective action has the form

$$
\bar{\Gamma}^{(1)}=-\frac{i}{2} \int d^{8} z \int_{0}^{\infty} \frac{d s}{s}<A(\tilde{s})+\tilde{A}(\tilde{s})>\left.U\left(x, x^{\prime} ; s\right)\right|_{x=x^{\prime}}
$$

where $A(\tilde{s})$ and $\tilde{A}(\tilde{s})$ are given by $(28)$. We will calculate

$$
<A(\tilde{s})+\tilde{A}(\tilde{s})>\left.U\left(x, x^{\prime} ; s\right)\right|_{x=x^{\prime}}
$$

One can represent this expression in the form

$$
<A(\tilde{s})+\tilde{A}(\tilde{s})>U\left(x, x^{\prime} ; s\right)=\sum_{n=0}^{\infty} f_{n}(\tilde{s}) \square^{n} U\left(x, x^{\prime} ; s\right)
$$

Here $U\left(x, x^{\prime} ; s\right)=\exp \left(i(s+i \epsilon) \square^{2}\right) \delta^{4}\left(x-x^{\prime}\right)$. Our aim is to compute $\left.\square^{n} U(x, x ; s)\right|_{x=x^{\prime}}$. Using the Fourier representation

$$
U\left(x, x^{\prime} ; s\right)=\int \frac{d^{4} k}{(2 \pi)^{4}} e^{i(s+i \epsilon) k^{4}-i k\left(x-x^{\prime}\right)},
$$

ones obtain

$$
\square^{n} U\left(x, x^{\prime} ; s\right)=\int \frac{d^{4} k}{(2 \pi)^{4}}\left(-k^{2}\right)^{n} e^{i(s+i \epsilon) k^{4}-i k\left(x-x^{\prime}\right)}
$$

When $x=x^{\prime}$ we find

$$
\left.\square^{n} U\left(x, x^{\prime} ; s\right)\right|_{x=x^{\prime}}=\int \frac{d^{4} k}{(2 \pi)^{4}}\left(-k^{2}\right)^{n} e^{i(s+i \epsilon) k^{4}}
$$

If $n$ is even, $n=2 l$, then

$$
\square^{n} U(x, x ; s)=\left(-i \frac{\partial}{\partial s}\right)^{l} \int \frac{d^{4} k}{(2 \pi)^{4}} e^{i(s+i \epsilon) k^{4}}
$$

if $n=2 l+1$ then

$$
\square^{n} U(x, x ; s)=\left(-i \frac{\partial}{\partial s}\right)^{l} \int \frac{d^{4} k}{(2 \pi)^{4}}\left(-k^{2}\right) e^{i(s+i \epsilon) k^{4}}
$$

where we take into account

$$
\square^{2} U=-i \frac{\partial}{\partial s} U
$$

For the integrals arisen one obtains

$$
\begin{array}{r}
\int \frac{d^{4} k}{(2 \pi)^{4}} e^{i(s+i \epsilon) k^{4}}=\frac{1}{32 \pi^{2} \tilde{s}} \\
\int \frac{d^{4} k}{(2 \pi)^{4}}\left(-k^{2}\right) e^{i(s+i \epsilon) k^{4}}=\frac{-\sqrt{\pi}}{32 \pi^{2} \widetilde{s}^{3 / 2}}
\end{array}
$$


where $\tilde{s}=-i(s+i \epsilon)$. The $<A(\tilde{s})+\tilde{A}(\tilde{s})>$ due to 29 can be written as

$$
<A(\tilde{s})+\tilde{A}(\tilde{s})>=2 \sum_{n=0}^{\infty} \frac{\left(4 \tilde{s} \sqrt{h \bar{h} \varphi \bar{\varphi}}^{2 n+2}\right.}{(2 n+2) !} \square^{n}
$$

After using (A.II) and (A.III) and carrying out the Wick rotation we obtain the contribution to the effective action corresponding to general effective potential in the form

$$
\begin{aligned}
K^{(1)} & =\int d^{8} z \int_{0}^{\infty} \frac{d \tilde{s}}{\tilde{s}} \sum_{l=0}^{\infty}\left[\left.\frac{(4 \sqrt{h \bar{h}} \tilde{s})^{4 l+2}}{(4 l+2) !}(\varphi \bar{\varphi})^{2 l+1} \square^{2 l} U\right|_{x=x^{\prime}}+\right. \\
& \left.+\left.\frac{(4 \sqrt{h \bar{h}} \tilde{s})^{4 l+4}}{(4 l+4) !}(\varphi \bar{\varphi})^{2 l+2} \square^{2 l+1} U\right|_{x=x^{\prime}}\right]
\end{aligned}
$$

Or in the equivalent form

$$
\begin{aligned}
K^{(1)} & =\int d^{8} z \int_{0}^{\infty} d \tilde{s} \sum_{l=0}^{\infty}\left[\frac{(16 h \bar{h} \varphi \bar{\varphi}))^{2 l+1}}{(4 l+2) !} \frac{(-1)^{l} l !}{32 \pi^{2}} \tilde{s}^{3 l}-\right. \\
& \left.-\frac{(16 h \bar{h} \varphi \bar{\varphi}))^{2 l+2}}{(4 l+4) !} \frac{(-1)^{l+1}(2 l+1) ! !}{32 \pi^{3 / 2}} \tilde{s}^{3 l+3 / 2}\right]
\end{aligned}
$$

Now let us put $u=(16 h \bar{h} \varphi \bar{\varphi})^{2 / 3} \tilde{s}$. It leads to the expression

$$
\begin{aligned}
K^{(1)} & =\Lambda^{1 / 3} \bar{\Lambda}^{1 / 3}\left(\frac{(4 \pi)^{2}}{Q^{2}}\right)^{2 / 3} \int d^{8} z e^{\sigma+\bar{\sigma}} \times \\
& \times \int_{0}^{\infty} d u \sum_{l=0}^{\infty}\left(\frac{u^{3 l}(-1)^{l} l !}{32 \pi^{2}(4 l+2) !}-\frac{u^{3 l+3 / 2}(-1)^{l}(2 l+1) ! !}{32 \pi^{3 / 2} 2^{l}(4 l+4) !}\right)
\end{aligned}
$$

(we have restored $\Lambda$ and $\varphi$ using $h=-36 \frac{\pi^{2} \Lambda}{Q^{2}}, \varphi \bar{\varphi}=e^{3(\sigma+\bar{\sigma})}$ ) The contribution to general effective action has the form

$$
K^{(1)}=\frac{c}{Q^{4 / 3}}|\Lambda|^{2 / 3} \int d^{8} z e^{\sigma+\bar{\sigma}}
$$

where $c$ is a positive finite constant. Namely,

$$
c=(4 \pi)^{4 / 3} \int_{0}^{\infty} d u \sum_{l=0}^{\infty}\left(\frac{u^{3 l}(-1)^{l} l !}{32 \pi^{2}(4 l+2) !}-\frac{u^{3 l+3 / 2}(-1)^{l}(2 l+1) ! !}{32 \pi^{3 / 2} 2^{l}(4 l+4) !}\right)
$$

The proof of finiteness of this constant is analogous with the proof of finiteness of constants arisen when calculating chiral effective action which will be carried out in Appendix B. 


\section{Appendix B. Calculation of one-loop chiral effective la-} grangian

Due to (34) we can represent $A+\tilde{A}$ in the form

$$
\begin{aligned}
A+\tilde{A} & =512 h \bar{h} \varphi \tilde{s}^{2}+\sum_{l=0}^{\infty}\left\{\frac{\left(16^{2} h \bar{h}\right)^{l+1} \varphi^{l+1}}{(2 l+2) !} \tilde{s}^{2 l+2}+9 \Lambda \frac{\left(16^{2} h \bar{h}\right)^{l+1} \varphi^{l}}{(2 l+3) !} \times\right. \\
& \left.\times \tilde{s}^{2 l+3} D^{2} \varphi+2^{21} h \bar{h}^{2} \frac{D^{\alpha} \varphi D_{\alpha} \varphi}{\varphi}\left(16^{2} h \bar{h}\right)^{l} \varphi^{l} \tilde{s}^{2 l+3}\left(\frac{1}{(2 l+2) !}-\frac{3}{(2 l+3) !}\right)\right\} \square^{l}
\end{aligned}
$$

Now we are again use the rule (A.I) and (A.II) and carry out the Wick rotation. Transforming (A.I) to the form of the integral over chiral subspace we arrive at

$$
\begin{aligned}
\mathcal{L}_{c}^{(1)} & =\frac{1}{2} \int_{0}^{\infty} d \tilde{s}\left(-\frac{1}{4} \bar{D}^{2}\right) \sum_{k=0}^{\infty}\left\{-\left[4 h \bar{h}^{2} \frac{\left(16^{2} h \bar{h}\right)^{2 k} \varphi^{2 k}}{(4 k+3) !} D^{2} \varphi+8^{3} h \bar{h}^{2}\left(16^{2} h \bar{h}\right)^{2 k} \varphi^{2 k-1} \times\right.\right. \\
& \left.\times\left(\frac{1}{(4 k+2) !}-\frac{3}{(4 k+3) !}\right) D^{\alpha} \varphi D_{\alpha} \varphi\right] \frac{(-1)^{k} k !}{32 \pi^{2}} \tilde{s}^{3 k+1}+ \\
& +\left[4 h \bar{h}^{2} \frac{\left(16^{2} h \bar{h}\right)^{2 k} \varphi^{2 k}}{(4 k+5) !} D^{2} \varphi+8^{3} h \bar{h}^{2}\left(16^{2} h \bar{h}\right)^{2 k+1} \varphi^{2 k+1} \times\right. \\
& \left.\left.\times\left(\frac{1}{(4 k+4) !}-\frac{3}{(4 k+5) !}\right) D^{\alpha} \varphi D_{\alpha} \varphi\right] \frac{(-1)^{k}(2 k+1) ! !}{2^{k} 32 \pi^{3 / 2}} \tilde{s}^{3 k+5 / 2}\right\}
\end{aligned}
$$

Terms of the form $\bar{D}^{2} \Phi^{n}$ are omitted here since they are evidently equal to zero. Let us put $u=(16 \sqrt{h \bar{h}})^{4 / 3} \varphi^{2 / 3} \tilde{s}$. Then we obtain

$$
\begin{aligned}
\mathcal{L}_{c}^{(1)} & =\frac{1}{2} \int_{0}^{\infty} d u\left(-\frac{1}{4} \bar{D}^{2}\right) \frac{1}{(16 \sqrt{h \bar{h}})^{4 / 3} \varphi^{2 / 3}} \sum_{k=0}^{\infty} u^{3 k}\left\{-\left[4 h \bar{h}^{2} \frac{1}{(4 k+3) !} \times\right.\right. \\
& \left.\times D^{2} \varphi+8^{3} h \bar{h}^{2} \varphi^{-1}\left(\frac{1}{(4 k+2) !}-\frac{3}{(4 k+3) !}\right) D^{\alpha} \varphi D_{\alpha} \varphi\right] \times \\
& \times \frac{(-1)^{k} k !}{32 \pi^{2}} \frac{u}{(16 \sqrt{h \bar{h}})^{4 / 3} \varphi^{2 / 3}}+\left[4 h \bar{h}^{2} \frac{1}{(4 k+5) !} D^{2} \varphi+8^{3} h \bar{h}^{2}\left(16^{2} h \bar{h}\right) \varphi \times\right. \\
& \left.\left.\times\left(\frac{1}{(4 k+4) !}-\frac{3}{(4 k+5) !}\right) D^{\alpha} \varphi D_{\alpha} \varphi\right] \frac{(-1)^{k}(2 k+1) ! !}{2^{k} 32 \pi^{3 / 2}} \frac{u^{5 / 2}}{(16 \sqrt{h \bar{h}})^{10 / 3} \varphi^{5 / 3}}\right\}
\end{aligned}
$$

It is equal to

$$
\begin{aligned}
\mathcal{L}_{c}^{(1)} & =2 \int_{0}^{\infty} d u \sum_{k=0}^{\infty} u^{3 k}\left\{\partial_{m} \varphi \partial^{m} \varphi \times\right. \\
& \times\left[8^{3} h^{-1 / 3} \bar{h}^{2 / 3}\left(\frac{1}{(4 k+2) !}-\frac{3}{(4 k+3) !}\right) \frac{(-1)^{k} k !}{32 \pi^{2}} \frac{u}{\varphi^{7 / 3}}-\right. \\
& \left.-\left(\frac{1}{(4 k+4) !}-\frac{3}{(4 k+5) !}\right) \frac{(-1)^{k}(2 k+1) ! !}{2^{k} 32 \pi^{3 / 2}} h^{2 / 3} \bar{h}^{-1 / 3} \frac{u^{5 / 2}}{\varphi^{4 / 3}}\right]+ \\
& +\square \varphi\left[h^{-1 / 3} \bar{h}^{2 / 3} \frac{1}{(4 k+3) !} \frac{(-1)^{k} k !}{32 \pi^{2}} \frac{u}{\varphi^{4 / 3}}-\right. \\
& \left.\left.-h^{-4 / 3} \bar{h}^{5 / 3} \frac{1}{(4 k+5) !} \frac{(-1)^{k}(2 k+1) ! !}{2^{k} 32 \pi^{3 / 2}} \frac{u^{5 / 2}}{\varphi^{7 / 3}}\right]\right\}
\end{aligned}
$$


The constants $c_{1}, c_{2}, c_{3}, c_{4}$ introduced in (35) have the form

$$
\begin{aligned}
& c_{1}=18 \int_{0}^{\infty} d u \sum_{k=0}^{\infty} u^{3 k+1} 8^{3}\left(\frac{1}{(4 k+2) !}-\frac{3}{(4 k+3) !}\right) \frac{(-1)^{k} k !}{32 \pi^{2}} \\
& c_{2}=-18 \int_{0}^{\infty} d u \sum_{k=0}^{\infty} u^{3 k+5 / 2}\left(\frac{1}{(4 k+4) !}-\frac{3}{(4 k+5) !}\right) \frac{(-1)^{k}(2 k+1) ! !}{2^{k} 32 \pi^{3 / 2}} \\
& c_{3}=6 \int_{0}^{\infty} d u \sum_{k=0}^{\infty} u^{3 k+1} \frac{1}{(4 k+3) !} \frac{(-1)^{k} k !}{32 \pi^{2}} \\
& c_{4}=-6 \int_{0}^{\infty} d u \sum_{k=0}^{\infty} u^{3 k+5 / 2} \frac{1}{(4 k+5) !} \frac{(-1)^{k}(2 k+1) ! !}{2^{k} 32 \pi^{3 / 2}}
\end{aligned}
$$

All these integrals over $u$ are finite. Let us prove this statement.

We start with the following integrals

$$
\begin{aligned}
& I_{1}=\int_{0}^{\infty} d u \sum_{k=0}^{\infty} u^{3 k+1} \frac{(-1)^{k} k !}{(4 k+2) !} \\
& I_{2}=\int_{0}^{\infty} d u \sum_{k=0}^{\infty} u^{3 k+5 / 2} \frac{(-1)^{k}(2 k+1) ! !}{2^{k}(4 k+4) !}
\end{aligned}
$$

Let us consider the identity

$$
\frac{(2 n+1) ! !}{2^{n}}=\frac{1}{\sqrt{\pi}} \Gamma\left(n+\frac{3}{2}\right)=\frac{1}{\sqrt{\pi}} \int_{0}^{\infty} d t e^{-t} t^{n+\frac{1}{2}}
$$

It leads to

$$
\begin{aligned}
& I_{1}=\int_{0}^{\infty} d u u \int_{0}^{\infty} d t e^{-t}\left(u^{3} t\right)^{-1 / 2} \sum_{k=0}^{\infty}\left(\left(u^{3} t\right)^{1 / 4}\right)^{4 k+2} \frac{(-1)^{k}}{(4 k+2) !} \\
& I_{2}=\frac{1}{2 \sqrt{\pi}} \int_{0}^{\infty} d u u \int_{0}^{\infty} d t e^{-t}\left(u^{3} t\right)^{-1 / 2} \sum_{k=0}^{\infty}\left(\left(u^{3} t\right)^{1 / 4}\right)^{4 k+4} \frac{(-1)^{k}}{(4 k+4) !}
\end{aligned}
$$

Let us use the following formulas

$$
\begin{aligned}
& \sum_{n=0}^{\infty} \frac{a^{4 n+2}}{(4 n+2) !}(-1)^{n}=-\frac{i}{2}\left(\cosh \frac{1+i}{\sqrt{2}} a-\cosh \frac{1-i}{\sqrt{2}} a\right) \\
& \sum_{n=0}^{\infty} \frac{a^{4 n+4}}{(4 n+4) !}(-1)^{n}=-\frac{1}{2}\left(\cosh \frac{1+i}{\sqrt{2}} a+\cosh \frac{1-i}{\sqrt{2}} a\right)+1
\end{aligned}
$$

As a result we obtain

$$
\begin{aligned}
I_{1} & =-\frac{i}{2} \int_{0}^{\infty} d u \int_{0}^{\infty} d t e^{-t}(u t)^{-1 / 2}\left(\cosh \frac{1+i}{\sqrt{2}}\left(u^{3} t\right)^{1 / 4}-\cosh \frac{1-i}{\sqrt{2}}\left(u^{3} t\right)^{1 / 4}\right) \\
I_{2} & =\frac{1}{2 \sqrt{\pi}} \int_{0}^{\infty} d u \int_{0}^{\infty} d t e^{-t}(u t)^{-1 / 2}\left(\left(-\frac{1}{2}\right) \times\right. \\
& \left.\times\left(\cosh \frac{1+i}{\sqrt{2}}\left(u^{3} t\right)^{1 / 4}+\cosh \frac{1-i}{\sqrt{2}}\left(u^{3} t\right)^{1 / 4}\right)+1\right)
\end{aligned}
$$

These integrals are evidently finite. The analogous consideration shows that the other intergals in eq. (B.IV) are also finite. 


\section{References}

[1] I.L.Buchbinder, A.Yu.Petrov. Class. Quant. Grav. 1996 (to be published); hepth 9511025 .

[2] I.L.Buchbinder, S.M.Kuzenko. Phys. Lett. B202 (1988), 233.

[3] I.L.Buchbinder, S.M.Kuzenko, J.V.Yarevskaya. Nucl. Phys. B411 (1992), 665

[4] I.L.Buchbinder, S.M.Kuzenko, J.V.Yarevskaya. Yad. Fiz. (Phys.Atom.Nucl.) 56 (1993), 202

[5] I.L.Buchbinder, S.M.Kuzenko. Ideas and Methods of Supersymmetry and Supergravity, IOP Publishing, Bristol and Philadelphia, 1995

[6] P.West. Phys. Lett. B258 (1991), 375.

[7] I.Jack, D.R.T.Jones, P.West. Phys. Lett. B258 (1991), 382.

[8] I.L.Buchbinder, S.M.Kuzenko, A.Yu.Petrov. Phys. Lett. B321 (1994), 372

[9] I.L.Buchbinder, S.M.Kuzenko, A.Yu.Petrov, J.V.Yarevskaya. Superfield Effective Potential, in Heat Kernel Techniques and Quantum Gravity, ed. by S.A.Fulling, Discourses in Mathematics and its Applications, No.4, Department of Mathematics, Texas A\&M University, College Station, Texas, 1995, pp. 151-161.

[10] I.L.Buchbinder, S.M.Kuzenko, A.Yu.Petrov. Yad.Fiz. (Phys.Atom.Nucl.) 59 (1996), 167

[11] N.Seiberg. Phys. Lett. B206 (1988), 75.

[12] N.Seiberg. Phys. Lett. B318 (1993), 469.

[13] I.L.Buchbinder, S.D.Odintsov, I.L.Shapiro. Effective Action in Quantum Gravity, IOP, Bristol and Philadelphia, 1992.

[14] I.Antoniadis, E.Mottola. Phys. Rev. D45 (1992), 2013.

[15] E.Elizalde, S.D.Odintsov. Phys. Lett. B315 (1993), 245.

[16] E.Elizalde, S.D.Odintsov. Mod. Phys. Lett. A8 (1993), 3325.

[17] E.Elizalde, S.D.Odintsov. Phys. Lett. B334 (1994), 33.

[18] S.D.Odintsov. Z.Phys. C54, 531 (1992).

[19] I.Antoniadis and S.D.Odintsov. Phys.Lett. B343, 76 (1995).

[20] I.L.Shapiro. Mod. Phys. Lett. A9 (1994), 1985.

[21] I.L.Shapiro, G.Cognola. Phys. Rev. D51 (1995), 2775.

[22] I.L.Shapiro, H.Takata. Phys. Rev. D52 (1995), 2162.

[23] S.J.Gates. Nucl. Phys. B238 (1984), 349. 